\title{
The Use of Music in a Graphical Interface for the Visually Impaired.
}

\author{
Dimitrios I. Rigas and James L. Alty \\ LUTCHI Research Centre \\ Dept. of Computer Studies \\ Loughborough University \\ Loughborough \\ Leicestershire LE11 3TU, UK \\ d.rigas@lboro.ac.uk and j.1.alty@lboro.ac.uk
}

\begin{abstract}
The possibility of using music to communicate information of a spatial nature to visually impaired users is explored. The development of a tool - the AUDIOGRAPH - which enables visually impaired users to appreciate and manipulate objects contained in a graphical area is described. Early results show that the musical metaphors used did successfully communicate the general spatial layout and the types of objects contained in the space. Music was also successfully used to control the interface.
\end{abstract}

KEY WORDS: Interface Design, Visually Impaired Users, Music, Graphics, Auditory Interfaces.

\section{Introduction}

Graphical User Interfaces (or GUIs as they are often known) are now ubiquitous. They are offered as UserFriendly interfaces with a high utility. Whilst this is almost certainly true, resulting in better usability for a much wider user base, they also represent a serious step backwards for blind or visually impaired users. Traditionally, blind users have been successful in exploiting computer technology using devices such as Screen Readers to replace the visual sense. However, Screen Readers are not effective when applied to Graphical Interfaces, and this fact has begun to seriously limit visually impaired use.

The communication of graphical information to blind users is difficult to achieve using speech. The intensely serial nature of the speech medium, together with its language basis, requires continual translation and is very slow and error prone. We therefore decided to explore an alternative auditory medium which could suggest graphical shapes directly in some way. The most highly structured auditory medium being music, we expected that a study into the use of music for conveying graphical information could be fruitful.

\section{Auditory Interfaces}

The use of audio in human computer interfaces can be traced back to Bly (1982). Gaver (1986) later proposed the use of Auditory Icons and used them in the SonicFinder (1989) to assist visually impaired users with editing. Auditory Icons, however, are short bursts of familiar sounds rather than music. Edwards (1989) developed the Soundtrack system to assist blind users in editing, but again the use of music was very limited. Blattner et al, (1989) moved neared to exploiting music by proposing the use of Earcons - short musical melodies which share a common structure such as rhythm. Blattner also used the properties of musical structures to communicate the flow in a turbulent liquid, interestingly, utilising musical compositional techniques which were 
hundreds of years old. Brown and Hershberger (1992) used music and sound to support the visualisation of Sorting Algorithms, and Alty (1995) has developed purely musical mappings for the Bubble Sort and the Minimum Path Algorithm. Recently some progress has been made in assisting program debugging using music, for example, Bock (1995), and Vickers and Alty (1996). However there are very few recorded experiments to evaluate the use of music in human computer interaction.

\section{Rationale behind the Investigations}

The main objective behind the set of experiments reported in this paper, was to determine if blind users could, by the use of music alone, appreciate the spatial layout of objects in a graphical area and successfully perform a set of graphical operations on them using musical feedback. Because of the emphasis on music, and the fact that there was little previous work on the use or evaluation of musical representations, we decided to use music alone to communicate all information about the interface. We appreciate that in a real interface, such as a commercial tool, it would be sensible to use speech in addition to music (particularly for conveying exact information), but we felt that the inclusion of other auditory modes of interaction would risk confusion in the interpretation of the experimental results.

\section{The AUDIOGRAPH system}

We have therefore constructed an experimental prototype tool on which to carry out the investigations into the use of music in a graphical tool, (called the AUDIOGRAPH). AUDIOGRAPH has both a visual interface (for the experimenter) and a completely auditory interface for blind users. Because of the graphical nature of the domain it is easiest to describe it in terms of its visual interface first.

Input interaction with the system is exclusively via the keyboard. This mode was chosen after extensive discussions with blind users who pointed out the mouse has a number of disadvantages for blind and visually impaired users. The arrow keys are used for moving the cursor round the area, and other keys (usually any key) are used to confirm actions. All musical output is produced by MIDI output from Visual Basic, communicated to a stereo sound system using a Creative Labs Soundblaster 16-bit card. The actual sounds were created using a Roland MT32 Multi-timbre output device. All output information was communicated using music alone.

It is important to note that the AUDIOGRAPH tool was aimed at the average user (from a musical ability point of view). No special musical ability was expected for any of the experiments performed. A musical questionnaire was given to all participants to check their musical experience and knowledge.

\section{Musical Mappings}

A number of different types of information need to be communicated

1. the current position of the cursor or a graphical object

2. the nature of a graphical object (e.g. its type, size and shape)

3. the selection and application of graphical operations

4. the occupancy of the space around the cursor

5. file names for storage and retrieval

Furthermore it was postulated that user interaction would be best supported by a consistent use of music, that is, similar metaphors would be used over a range of domain items.

One of the most important aspects to communicate is that of spatial position. It was considered that a mapping from a number into pitch would provide a reasonably good mapping. This mapping had been used in the Bubble Sort of Alty (1995), where good correlations had been obtained between number and pitch. It is well known that users cannot achieve a completely accurate mapping to pitch, and problems have been reported in the use of intervals greater than an octave Deutch (1982). However, the objective in AUDIOGRAPH is not to communicate accurate positions but rather, a good sense of relative positions in the graphical space. Furthermore, the horizontal and vertical co-ordinates needed to be communicated separately and be easily identified. It was decided to use separation in time (i.e. the $\mathrm{X}$ co-ordinate would always be communicated before the $Y$ co-ordinate, and the two would be communicated serially with a short pause inbetween). We felt that some additional redundancy would also be helpful and expected that timbre would assist in this respect (i.e. use of different 
instruments for $X$ and $Y$ ). However the use of timbre is potentially problematic. If a user is told that the $X$ coordinate is played on a trumpet, and the $\mathrm{Y}$ co-ordinate on a Piano, can the average musically untrained user successfully identify each instrument?

\subsection{Identification of the Co-ordinate using Timbre}

Users were first asked to name the instruments which they felt they could recognise.

\begin{tabular}{llll|}
\hline $\begin{array}{l}\text { Musical } \\
\text { Instrument }\end{array}$ & $\begin{array}{l}\text { \% of } \\
\text { Confidence }\end{array}$ & $\begin{array}{l}\text { Musical } \\
\text { Instrument }\end{array}$ & $\begin{array}{l}\text { \% of } \\
\text { Confidence }\end{array}$ \\
\hline Guitar & 96 & Xylophone & 30 \\
Piano & 94 & Trombone & 39 \\
Timpani & 83 & Pan Pipes & 27 \\
Violin & 82 & Tuba & 20 \\
Saxophone & 76 & Oboe & 17 \\
Flute & 55 & Piccolo & 16 \\
Harp & 53 & French Horn & 13 \\
Trumpet & 42 & Bassoon & 10 \\
Castanets & 40 & Celeste & 3 \\
Cello & 37 & Contrabass & 3 \\
Harmonica & 37 & Harpsichord & 2 \\
Organ & 35 & Mandolin & 1 \\
Clarinet & 34 & Cor Anglais & 1 \\
\hline
\end{tabular}

Table 1 Instrumental Timbres which Users thought they could identify

There are some instruments which almost all users thought they could identify (for example, Piano, Timpani and Guitar) whereas other instruments had very poor ratings (e.g. Mandolin, Harpsichord and Celeste - though this is probably because they did not associate the name with the sound). There was no significant difference between the sighted and blind users.

\begin{tabular}{|l|llllll|}
\hline & Piano & Wind & Timp & Brass & Strings & Organ \\
\hline Piano & 65 & 0 & 0 & 2 & 3 & 4 \\
Wind & 4 & 59 & 0 & 5 & 13 & 15 \\
Timp & 0 & 0 & 15 & 0 & 0 & 0 \\
Brass & 8 & 7 & 0 & 18 & 11 & 4 \\
Strings & 2 & 9 & 0 & 5 & 14 & 2 \\
Organ & 1 & 4 & 0 & 3 & 5 & 15 \\
\hline
\end{tabular}

Table 2 Users actual Timbre recognition performance
Then, all the instrumental timbres in the list were played to subjects, who then had to assign them to named instruments. A truncated form of the results is shown in Table 2 and are interesting because they do not always reflect the users' anticipations in Table 1.

The instruments in Table 2 have been grouped according to their conventional musical families (strings, woodwind, brass and drums), though we have departed from this classification by grouping Piano, Celeste, Xylophone and Guitar as one family (plucked or hit instruments) since the average user would not group Piano and Xylophone with Percussion. Similarly we have grouped Organ and Harmonica together. Certain instruments were readily recognised (Piano, Organ, Guitar, Flute, Drums etc..) and confusion within families was more common than between families. This suggests that instruments such as Piano and Organ can easily be distinguished by most non-musicians, but that designers should avoid the expectation that such users can distinguish timbre within musical families (e.g. a Cello and a Violin).

We therefore decided to use the Piano for the horizontal co-ordinate and the Organ for the vertical co-ordinate.

\subsection{Communication of the Co-ordinates using Pitch}

The next question to be solved was how to communicate the length of the co-ordinate. We had already docided to use pitch, but a number of options were open to us.

1. Use the Chromatic or Diatonic scale. The former provides more intervals, but the latter may be more familiar.

2. Using a particular note as origin, play all the notes between the origin and the value required. This has the advantage of communicating length both through pitch and time. We call this the Note-Sequence technique.

3. Play only the origin and the final value note. This has the advantage of brevity, but may run into problems because of octaves. We call this the NotePair technique.

A set of experiments was carried out with 12 blind users for all three conditions. In every case the range was 40 
notes. No users in the sample were professional musicians. The experimental procedures were as follows:

1. the scale (Diatonic or Chromatic) was sounded over the 40 notes. Then the $\mathrm{X}$ co-ordinate was sounded, followed by a pause. Then the $\mathrm{Y}$ co-ordinate was sounded.. The user was asked to identify the coordinate being communicated. The order of presentation of the co-ordinates was randomised.

2. the experiments were carried out in order, ChromaticSequence, Diatonic-sequence, Chromatic pair, Diatonic Pair.

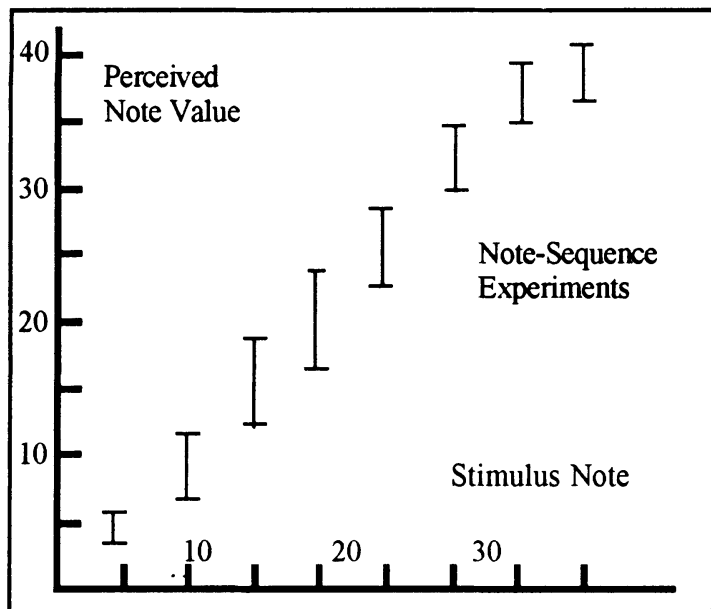

Figure 1(a) Means and Standard Deviations for the Note Sequence Results

The stimulus and response measures in Figures 1(a) and 1(b) are relative tone values based upon the $\mathrm{E}$ below middle $\mathrm{C}$. Thus, middle $\mathrm{C}$ corresponds to the number 9 in the Chromatic scale. Overall, there was no significant difference between the Chromatic Scale and the Diatonic Scale.

Figures 1(a) and 1 (b) show the variation of the means and standard deviations of the perceived co-ordinate against the stimulus, for both $\mathrm{X}$ and $\mathrm{Y}$ co-ordinates and for the Note-Sequence and Note-Pair experiments for the Chromatic scale. Thus there was expected to be some learning effect for the Note-Pair experiment.
The variance is much greater in the Note-Pair-Pair results than in the Note-Sequence results, and this is in spite of some learning which must have taken

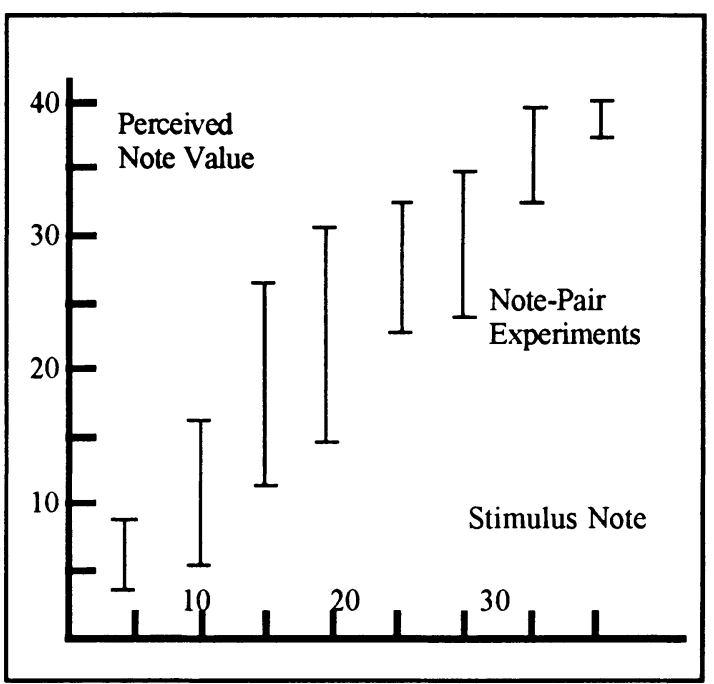

Figure 1(b) Means and Standard Deviations for the NotePairs results.

place because the Note-Pair experiment was second.

The difference between the two approaches can be seen in the global error table (which shows the error distribution independent of position) in Table 3.

\begin{tabular}{|lllll|}
\hline Interface & Correct & \pm 1 & \pm 2 & \pm 3 \\
\hline Note-Sequ. & $22.27 \%$ & $34.45 \%$ & $17.45 \%$ & $11.53 \%$ \\
Note-Pair & $5.6 \%$ & $12.66 \%$ & $15.38 \%$ & $10.89 \%$ \\
\hline
\end{tabular}

Table 3 The Global error distribution for the Note-

Sequence and Note-Pair experiments.

Thus $75 \%$ of users could locate a position to within \pm 2 on the $40 \times 40$ grid using the Note-Sequence method, but this figure dropped to about $34 \%$ for the Note-Pair method, and this is in spite of increased exposure to the use of music. The figure of $74 \%$ for a \pm 2 error for the Note-Sequence method, (or $85 \%$ for \pm 3 error) is a good result, indicating that a user can obtain a general appreciation of the content of a graphical space using this technique, and the result is probably no worse than 
general visual memory for a similar task (people do not remember accurately the position of visual objects in a space).

Although Figure 1(a) shows that the location error in the Note-Sequence technique showed a steady worsening of performance as the co-ordinate value increased (there was an edge effect at the top end which arbitrarily reduced the errors there), performance was rarely worse than \pm 4 either way. As might be expected there was no difference in the performance with vertical and horizontal co-ordinates.

We conclude that blind users can use the Note-Sequence approach successfully to determine the co-ordinates of graphical objects to an accuracy acceptable for the general appreciation of space content. One reason why the approach works so well is that it uses three parallel mappings to communicate the co-ordinates - sequence length, counting of the notes, and pitch difference. Users can therefore trade-off between these three measures. It is also interesting to note that, with prolonged use, users became very adept at using the system.

\subsection{Cursor Control}

At first sight it might be thought that the Note-Sequence approach could not be used for cursor navigation because it would simply take too much time and this initially suggested that the less reliable Note-Pair technique would have to be used. However, it turns out that we can still use the Note-Sequence technique for cursor navigation without the time penalty. With a visual cursor, cursor movement is always shown incrementally, rather than absolutely. The cursor is never returned to the origin before each movement. Thus the musical feedback for an audio cursor only needs to communicate the incremental changes as the cursor navigates. As this happens, users effectively hear the relative Note-Sequence technique between the initial and final cursor positions. Furthermore, the total movement will normally be less than, say, \pm 15 , so an accuracy, at least as good as, and often better than, the average obtained with co-ordinate location is achieved.

\subsection{Identifying Graphical Objects}

Graphical objects were also communicated using a spatial metaphor based on pitch. As far as possible the musical description was designed to have a one-to-one mapping with the graphical shape, and the mapping chosen was the same as the Note-Sequence mapping (used for both location and cursor navigation). This was expected to offer a number of advantages including the rapid generation of a mental model of the shape and consistency with the other three mappings simultaneously operating in the space-co-ordinate location, cursor navigation and control actions. The musical shapes communicated are shown in Figure 2.

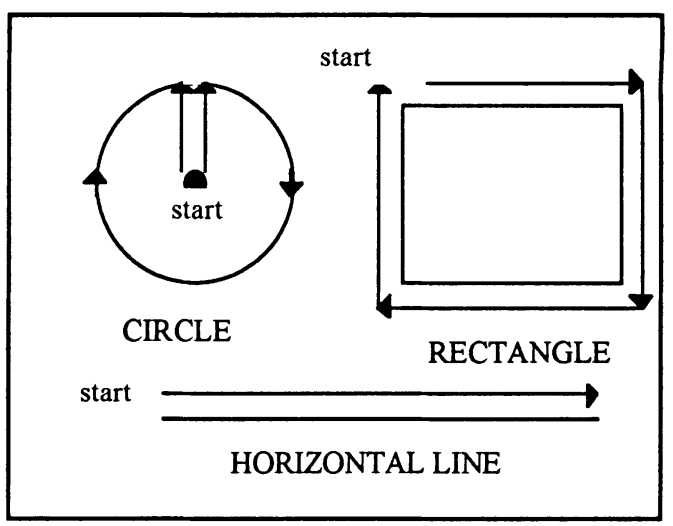

Figure 2 The Musical Description of Graphical Shapes

Without any prior training, $50 \%-60 \%$ of subjects were able to recognise the shapes without training (particularly the circle and the horizontal line). Even with very limited training, the recognition rate increased to nearly $100 \%$ for the Circle, and the Vertical and Horizontal lines, and to over $80 \%$ for the Rectangle and Square. With more training subjects could easily reach $100 \%$ recognition rates for all shapes.

With graphical objects it is also important to be able to obtain an indication of the size as well as the shape itself. The experimental results here were more variable. The overall means of the sizes of the various objects were reasonably accurate (so there was no systematic error as the size increased). However, the standard deviation varied with both the size and shape. Apart from the Circle, the error worsened with size. This is probably because, in general, the lengths being communicated are larger for the other objects (the Circle radius was communicated, not the diameter). Recognition and Size determination performance are good enough to allow 
qualitative measurements in the Graphical space to be made.

\subsection{Understanding the Musical Meanings of the Control Actions}

The three main editing operations (Contract, Expand and Undo) were communicated by Earcons. In these cases a specific meaning had to be communicated in a short time. Rather than use rhythmically based Earcons (as in the case of Blattner), the Earcons were constructed using musical patters which were expected to have strong semantic perceptual properties for the listener. Taking earlier work (e.g. Alty 1996, where users have been shown to be capable of recognising and drawing short tunes), compound Earcons were designed which communicated a change of shape over time signifying the control action. Thus, Expand consisted of a short tune which expanded in length from one to four items. Contract was the inverse of this. Undo consisted of a short rising tune with an "error" in it which was corrected in the second playing.

The results for Expand and Contract were astonishingly good. Most users picked up the meaning without needing to be prompted (of course they did know that the Earcon had to represent one of the three control actions). Undo was not recognised on the first pass, but became instantly recognisable once the underlying idea had been communicated. This latter point emphasises the importance of context in musical interaction. Once users had been told that the first section of Undo was a rising scale with an error, and that the second tune corrected it, they immediately understood.

\subsection{Other Facilities - Scanning}

AUDIOGRAPH also provided users with techniques for scanning the area, generally, and in the neighbourhood of the cursor, though the available space in this paper precludes a full description of the techniques and the results obtained. The techniques available were TopDown (scanning for the centre positions of objects by $\mathrm{X}$ and then by $\mathrm{Y}$ from the top of the diagram). Centre (scanning outwards from the cursor, locating objects centres as the spiral increased), and Ascending (scanning from the cursor to locate objects in ascending size). Users were able to successfully use the techniques to get a general impression of the contents of the graphical area.

\section{An Example Use of the AUDIOGRAPH}

To give an indication of how successful music was in conveying a diagram, blind users were presented with a number of diagrams and asked to "draw" out the content of the space. They used different scanning techniques to achieve this. An example diagram together with the user response is shown in Figures 3(a) and 3(b).

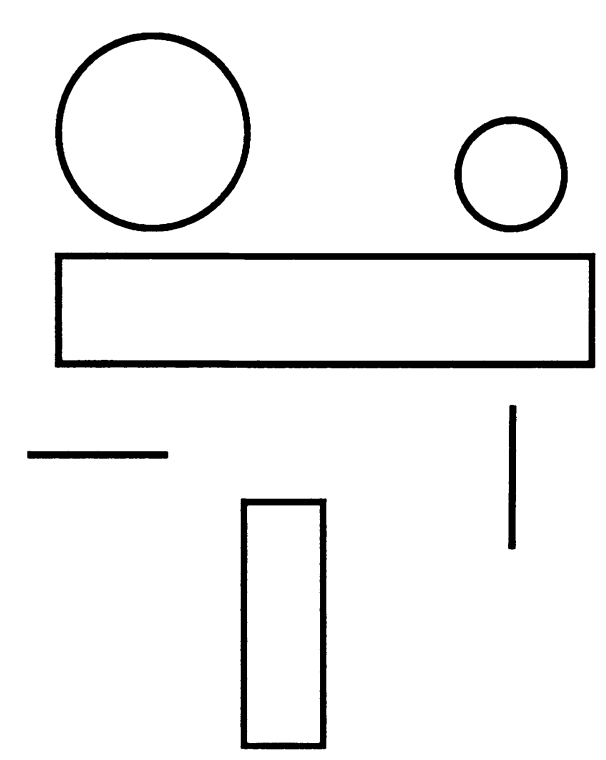

Figure 3 (a) The stimulus for a Blind User using AUDIOGRAPH who was required to interpret the diagram. 


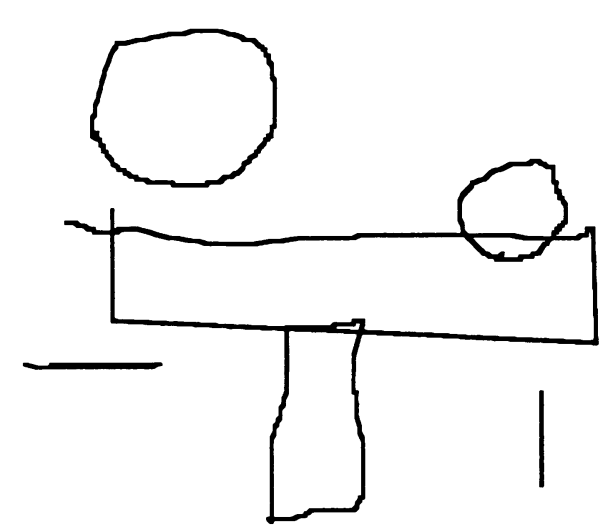

Figure 3 (b) User Perception of the diagram shown in 3(a) using the AUDIOGRAPH.

It should be appreciated that it is difficult for Blind users to accurately draw what they perceive. To assist them they were provided with paper which had a raised grid on it. However, the raised grid itself caused some deviations in the drawings. It can be seen that the user has perceived a successful overall view of the space.

Table 4 summarises the results from three drawn diagrams (from three different users) using the three different scanning methods. It details some comparative measures of how three diagrams were perceived, in terms of the number of shapes successfully reported, their relative sizes and an overall estimate of the spatial layout.

\begin{tabular}{|l|llll|}
\hline & $\begin{array}{l}\text { Centre } \\
\text { Scan }\end{array}$ & $\begin{array}{l}\text { Top } \\
\text { Down } \\
\text { Scan }\end{array}$ & $\begin{array}{l}\text { Ascend } \\
\text { Scan }\end{array}$ & actual \\
\hline No. Circles & 2 & 2 & 2 & 2 \\
No. Rects & 1 & 2 & 2 & 2 \\
No. Hor. lines & 3 & 1 & 1 & 1 \\
No. Ver. lines & 3 & 1 & 1 & 1 \\
Rel Size Circles & good & good & good & $2 ; 1$ \\
Rel Size Rects & good & good & fair & $3: 1$ \\
Spatial Overall & fair & good & good & \\
\hline
\end{tabular}

Table 4 Output from the same stimulus for three scanning methods
There were some discrepancies, for example, a subject in one example drew three lines (two Horizontal and one Vertical) which traced out three sides of the missing rectangle (line 2 of Table 4) so agreement was better than implied in Table 4. Agreement with the overall diagrams communicated (in terms of number of objects, relative size, and relative position) was quite good in most cases. It must be remembered that drawing on the raised paper was not easy and would contribute to errors. Some of the communicated diagrams were not so well perceived.

Although these results were satisfactory, it was felt that they might have been better given the results from the earlier location experiments. On reflection it was felt that the above experiment did not really represent the realworld situation, where most perceiving subjects would have some idea about the nature of the collection of objects being communicated.

In a second set of experiments in which a hint about the diagram ("Car", "Number", "Letter") was given, the importance of the perceptual context communicated was shown. When the subjects knew the diagram was a car, identification rose from $0 \%$ to $100 \%$. Similar results (though not always quite so dramatic) were obtained for the other contexts, for example an "E" shape. These results will be reported in detail, separately.

\section{Users Views of the Utility of the} AUDIOGRAPH.

Overall ratings for AUDIOGRAPH from blind users were $60 \%$ (Good or Very Good), with $30 \%$ (Average). Only $10 \%$ of users thought it was Poor. Users really liked the Graphical Drawing Area representation $(85 \%$ Good or Very Good), the Control Panel (85\% Good or Very Good), and the Auditory Cursor (85\% Good or Very Good). Less well thought of were, Sequential Pitch (35\% Good or Very Good, with $60 \%$ considering it Average), Graphical Object Description (65\% Good or Very Good and $25 \%$ Average) and Scanning (55\% Good or Very Good and 30\%Average), though all these were within acceptable levels. Most users pointed out the initial mappings were learned relatively easily and that any initial difficulties rapidly disappeared. 


\section{Conclusions}

Overall, the results from the AUDIOGRAPH are encouraging, both technically, and from a user point of view. Users could obtain a general "graphical" view of the space and interact with the system. Users, in general were pleasantly surprised by the effect of music (as opposed to speech). With a relatively short training period they were able to use the system successfully to locate, move and modify graphical objects. All users responded very positively when asked if music could be used for a graphical drawing tool, particularly in addition to speech. Only $20 \%$ responded that they would not wish to use music. Finally, all answered "yes" to the question "should this research continue to investigate music as a communication metaphor ?" However a number of limitations have come to light:

1. Listening to a reasonably complex diagram can take some time.

2. the support for communicating relationships between objects is poor

3. Memorability for large non-meaningful graphical spaces is low

From our observations of the interaction between the blind users and the system we wish to make three points about the use of music in the human computer interface. Firstly, with musical stimuli, context is everything. A musical stimulus can be interpreted in many ways, so providing users (blind or sighted) with the appropriate context in which to interpret the music is very important. Secondly, the use of multiple messages within one musical stimulus is also crucial. In our examples, the use of pitch, length and counting considerably aided comprehension. Thirdly, it is very important to design all musical messages within a consistent framework. In our work, the communication of co-ordinates, cursor position, graphical shape and scanning all used a similar metaphor, and we believe that this greatly aided overall comprehension.

\section{References}

Alty, J.L., (1996), "Can we use Music in HumanComputer Interaction?", in People and Computers X
(Proc. HCI'95), Kirby, M.A.R., Dix, A.J., and Finlay, J.E., (eds.), Cambridge Academic Press, pp 409 - 424.

Blattner, M.M, and Dannenberg, R.B., (1991), "Multimedia Interface Design", ACM Press, New York, Addison Wesley.

Blattner, M.M, Sumikawa, D.A., and Greenberg, R.M., (1989), "Earcons and Icons: Their Structure and Common Design Principles", in Human Computer Interaction, Vol. 4., pp 11 - 44.

Bly, S.A., (1982), “Communicating with Sound”, Proc. CHI82, ACM Press, Addison-Wesley NY, pp 371 - 375.

Bock, D.S., (1995), “Auditory Software diagnosis Using a Sound Domain Specification Language", Ph.D. Thesis, Syracuse university, Syracuse.

Brown, M., and Hershberger, J., (1992), "Colour and Sound in Algorithm Animation", IEEE Computer Vol. 25, No. 12, , pp 52 - 63.

Deutch, D., (1982), The Psychology of Music, Academic Press.

Edwards. A.D.N, (1989), "Soundtrack: An Auditory Interface for Blind Users", Human Computer Interaction, Vol. 4, No. 1, pp 45 - 66.

Gaver, W., (1986), "Using Sound in Computer Interfaces", Human Computer Interaction, Vol. 2, No. 2, pp $167-177$.

Gaver, W., (1989), "The SonicFinder: An Interface that Uses Auditory icons", Human Computer Interaction, Vol. 4, No.1, pp 87 - 94.

Vickers, P., and Alty, J.L., (1996), "CAITLIN: A Musical Program Auralisation Tool to Assist Novice Programmers with Debugging", in Proc. of ICAD'96, Santa Fe Institute, 1996. 